\title{
Resummation of Soft Gluon Logarithms in the DGLAP Evolution of Fragmentation Functions
}

\author{
S. Albino ${ }^{\top}$, B. A. Kniehl, and G. Kramer \\ II. Institut für Theoretische Physik, Universität Hamburg, \\ Luruper Chaussee 149, 22761 Hamburg, Germany \\ W. Ochs \\ Max-Planck-Institut für Physik (Werner-Heisenberg-Institut), \\ Föhringer Ring 6, 80805 München, Germany
}

(Dated: February 13, 2007)

\begin{abstract}
We define a general scheme for the evolution of fragmentation functions which resums both soft gluon logarithms and mass singularities in a consistent manner and to any order, and requires no additional theoretical assumptions. Using the Double Logarithmic Approximation and the known perturbative results for the splitting functions, we present our scheme with the complete contribution from the double logarithms, being the largest soft gluon logarithms. We show that the resulting approximation is more complete than the Modified Leading Logarithm Approximation even with the fixed order contribution calculated to leading order only, and find, after using it to fit quark and gluon fragmentation functions to experimental data, that this approximation in our scheme gives a good description of the data from the largest $x_{p}$ values to the peak region in $\xi=\ln \left(1 / x_{p}\right)$, in contrast to other approximations. In addition, we develop a treatment of hadron mass effects which gives additional improvements at large $\xi$.
\end{abstract}

PACS numbers: 12.38.Cy,12.39.St,13.66.Bc,13.87.Fh

\section{INTRODUCTION}

The current description of single hadron inclusive production processes within the parton model of perturbative QCD (pQCD) is provided by fragmentation functions (FFs) $D_{a}^{h}\left(x, Q^{2}\right)$, each of which corresponds at lowest order to the probability for the parton $a$ produced at short distance $1 / Q$ to form a jet that includes the hadron $h$ carrying a fraction $x$ of the longitudinal momentum of $a$. Different theoretical schemes have been derived depending on the kinematic region of $x$ : Fixed order (FO) calculations at intermediate and large $x$ and resummation to all orders of soft gluon logarithms (SGLs) at small $x$. What is needed is a single formalism valid over the union of all ranges that the different pQCD approaches allow. This unification must be consistent, i.e. it must agree with each approach in the set, when the expansion of that approach is used, up to the order being considered.

Much progress has been made 1, 2] in determining fragmentation functions (FFs) at large and intermediate momentum fraction $x$ using FO Dokshitzer-GribovLipatov-Altarelli-Parisi (DGLAP) evolution [3] to nextto-leading order (NLO) [4]. However, determination of FFs at small $x$ is performed independently, since the calculation of the evolution requires a different approach because of soft gluon logarithms (SGLs). The complete resummed contribution from the largest SGLs, being

"Current address: Institut für Theoretische Physik und Astrophysik, Universität Würzburg, 97074 Würzburg, Germany. the double logarithms (DLs), of the splitting functions is obtained from the Double Logarithmic Approximation (DLA) 5, 6], while some information on the contribution from the next largest class of SGLs, the single logarithms (SLs), in the splitting functions is obtained from the Modified Leading Logarithm Approximation (MLLA) 6, 7, 8]. (The complete evolution is obtained by using the approximation that the quark FFs are identical, and equal to the gluon FF multiplied by a calculable constant.) Provided all incomplete higher order terms are not allowed to become too large, the MLLA evolution can describe small $x$ data very well [9]. However, although the cross section over a large range of $x$ can be described by pQCD in general, two different approaches, for which no matching conditions exist, are required to relate one to the other. In addition, the range of $x$ over which both approaches are valid is not clear.

In this paper, we are concerned with the unification of DGLAP evolution and the resummation of SGLs in pQCD. In Sec. III we derive a simple and consistent scheme which reproduces both approaches to any desired order. In Sec. III] we use the DLA to obtain the complete DL contribution to the evolution within this scheme. These two sections justify and extend the scheme introduced in Ref. [10]. In Sec. IV] we compare this approximation with experimental data. Finally, in Sec. V] we present our conclusions. For the reader's convenience, we list the explicit results for the leading order (LO) splitting functions in the FO approach in Appendix A as well as references for the explicit results for the NLO ones. 


\section{SGL RESUMMATION IN DGLAP EVOLUTION}

In this section, we discuss DGLAP evolution and its SGLs in the FO approach, and then we give a formal definition of our general scheme in which the SGLs in this approach are resummed.

The DGLAP equation reads

$$
\frac{d}{d \ln Q^{2}} D\left(x, Q^{2}\right)=\int_{x}^{1} \frac{d y}{y} P\left(z, a_{s}\left(Q^{2}\right)\right) D\left(\frac{x}{y}, Q^{2}\right),
$$

where, for brevity, we omit hadron and parton labels. $D$ is a vector containing the gluon FF $D_{g}$ and the quark and antiquark FFs $D_{q}$ and $D_{\bar{q}}$ respectively, in linear combinations according to the choice of basis, and $P$ is the matrix of the splitting functions. We define $a_{s}=\alpha_{s} /(2 \pi)$, whose $Q^{2}$ dependence is determined by the QCD $\beta$ function $\beta\left(a_{s}\left(Q^{2}\right)\right)$, through the Callan-Symanzik equation

$$
\frac{d}{d \ln Q^{2}} a_{s}\left(Q^{2}\right)=\beta\left(a_{s}\left(Q^{2}\right)\right)
$$

The $\beta$ function can be calculated in perturbation theory, where it takes the form

$$
\beta\left(a_{s}\right)=-\sum_{n=2}^{\infty} \beta_{n-2} a_{s}^{n} .
$$

Choosing a factorization scheme in which $P$ is explicitly independent of quark masses and then exploiting the resulting $\mathrm{SU}\left(n_{f}\right)$ symmetry for $n_{f}$ quark flavours, and also exploiting the charge conjugation invariance in $P$, leads to the simplest basis for writing Eq. (1), which consists of the combinations (i) $D=D_{q}^{-}$, where $D_{q}^{-}=D_{q}-D_{\bar{q}}$ is the valence quark FF, (ii) $D=D_{N S}$, where $D_{N S}$ is a non-singlet quark FF, i.e. any linear combination of the FFs $D_{q}^{+}=D_{q}+D_{\bar{q}}$ which vanishes when they are all equal, and (iii) $D=\left(D_{\Sigma}, D_{g}\right)$, where

$$
D_{\Sigma}=\frac{1}{n_{f}} \sum_{q=1}^{n_{f}} D_{q}^{+}
$$

is the singlet quark FF. The $n_{f}-1$ non-singlets must be chosen such that together with the singlet a linearly independent basis for the quarks is formed.

We will often work in Mellin space, where a function $f(x)$ becomes

$$
f(\omega)=\int_{0}^{1} d x x^{\omega} f(x)
$$

where $\omega$ is any integer greater than those values of $\omega$ for which $f(\omega)$ is non-singular, since the convolution in $x$ space in Eq. (1), and in equations later in this paper, becomes the simple product

$$
\frac{d}{d \ln Q^{2}} D\left(\omega, Q^{2}\right)=P\left(\omega, a_{s}\left(Q^{2}\right)\right) D\left(\omega, Q^{2}\right) .
$$

After performing the desired analytic operations, Mellin space results can be transformed back to $x$ space by analytically continuing $f(\omega)$ to complex $\omega$ and then using the inversion formula

$$
f(x)=\frac{1}{2 \pi i} \int_{C} d \omega x^{-\omega-1} f(\omega),
$$

where $C$ is a contour in Mellin space from $\operatorname{Im}(\omega)=-\infty$ to $\operatorname{Im}(\omega)=\infty$, which passes to the right of all poles in $f(\omega)$.

Without knowledge of $P$, Eq. (1) (or Eq. (6)) provides no constraint on $D\left(x, Q^{2}\right)$ over the ranges $0 \leq x \leq 1$ and $0 \leq Q^{2} \leq \infty$. For a given $\omega$, specification of $P\left(\omega, Q^{2}\right)$ for all $Q^{2}$ fixes the the $Q^{2}$ evolution of $D\left(\omega, Q^{2}\right)$. This means that, given $D\left(\omega, Q_{0}^{2}\right)$ at some specified $Q_{0}^{2}, D\left(\omega, Q^{2}\right)$ can be calculated for all $Q^{2}$. This evolution is usually calculated explicitly for the evolution matrix $E$, defined for all $Q^{2}$ and $Q_{0}^{2}$ by

$$
D\left(\omega, Q^{2}\right)=E\left(\omega, a_{s}\left(Q^{2}\right), a_{s}\left(Q_{0}^{2}\right)\right) D\left(\omega, Q_{0}^{2}\right),
$$

and for all $a_{s}, a_{1}$ and $a_{0}$ by

$$
\begin{aligned}
E\left(\omega, a_{s}, a_{s}\right) & =I, \\
E\left(\omega, a_{s}, a_{1}\right) E\left(\omega, a_{1}, a_{0}\right) & =E\left(\omega, a_{s}, a_{0}\right),
\end{aligned}
$$

( $I$ is the unit matrix) with no loss of generality for the functional form of $D\left(\omega, Q^{2}\right)$. Then, with some additional definitions for $E$ that result in no loss of generality, $E$ becomes fully constrained in terms of $P$ by invoking Eq. (6),

$$
P\left(\omega, a_{s}\left(Q^{2}\right)\right)=\frac{d E\left(\omega, a_{s}\left(Q^{2}\right), a_{0}\right)}{d \ln Q^{2}} E^{-1}\left(\omega, a_{s}\left(Q^{2}\right), a_{0}\right) .
$$

The boundary conditions in Eq. (9) can be used to verify that the right hand side of Eq. (10) is independent of $a_{0}$.

The factorization theorem [11] states that $P$ is an invariant with respect to the hadron being observed, and furthermore that the series for $P\left(x, a_{s}\right)$ in $a_{s}$ keeping $x$ fixed,

$$
P\left(x, a_{s}\right)=\sum_{n=1}^{\infty} a_{s}^{n} P^{(n-1)}(x),
$$

can be calculated from perturbation theory even when any quark masses go to zero or infinity. Equation (11) truncated at some chosen (finite) $n$ is known as the FO approach, and is not valid at small $x$ due to the presence of terms which in the limit $x \rightarrow 0$ behave like $\left(a_{s}^{n} / x\right) \ln ^{2 n-m-1} x$ for $m=1, \ldots, 2 n-1$. Such logarithms are called SGLs, and $m$ labels their class. As $x$ decreases, these unresummed SGLs will spoil the convergence of the FO series for $P\left(x, a_{s}\right)$ once $\ln (1 / x)=O\left(a_{s}^{-1 / 2}\right)$. Consequently the evolution of $D\left(x, Q^{2}\right)$ will not be valid here, since the whole range $x \leq y \leq 1$ contributes in Eq. (11). Therefore, the FO approach is only a good approximation for sufficiently large $x$. 
SGLs are defined to be all those terms of the form $a_{s}^{n} / \omega^{2 n-m}$ only, where $m=1, \ldots, 2 n$ and labels the class of the SGL, in the expansion about $\omega=0$ of the Mellin transform of Eq. (11),

$$
P\left(\omega, a_{s}\right)=\sum_{n=1}^{\infty} a_{s}^{n} P^{(n-1)}(\omega) .
$$

We will consider SGLs of the type $m=2 n$ later. For $m=1, \ldots, 2 n-1$, this definition agrees with the form of the SGLs in $x$ space given above, since

$$
\frac{1}{\omega^{p}}=-\frac{(-1)^{p}}{p !} \int_{0}^{1} d x x^{\omega} \frac{\ln ^{p-1} x}{x}
$$

for $\operatorname{Re}(\omega)>0$ and $p \geq 1$. Such terms spoil the convergence of the series in Eq. (12) as $\omega \rightarrow 0$. What we require is an alternative scheme for the evolution which will be valid for large and small $\omega$. The inverse Mellin transform of this evolution should then be valid for large and small $x$. For this purpose, we propose the following general scheme, which we call the SGL+FO scheme. Firstly, $P$ is written in the form

$$
P=P^{\mathrm{FO}}+P^{\mathrm{SGL}},
$$

where $P^{\mathrm{SGL}}$ contains only and all the SGLs in $P$, so that $P^{\mathrm{FO}}$ is completely free of SGLs. Secondly, by summing all SGLs in each class $m, P^{\mathrm{SGL}}\left(\omega, a_{s}\right)$ is resummed in the form

$$
P^{\mathrm{SGL}}\left(\omega, a_{s}\right)=\sum_{m=1}^{\infty}\left(\frac{a_{s}}{\omega}\right)^{m} g_{m}\left(\frac{a_{s}}{\omega^{2}}\right)
$$

and truncated for some finite $m$. The functions $g_{m}(x)$ in Eq. (15) are not Taylor series in either $x$ or any function thereof. Note that, apart from the condition that the series must start at $m=1$, which follows from the definition of SGLs above, Eq. (15) is just the general result of expanding a function of $a_{s}$ and $\omega$ in $a_{s} / \omega$ keeping $a_{s} / \omega^{2}$ fixed. Thirdly, the remaining FO contribution to $P, P^{\mathrm{FO}}\left(\omega, a_{s}\right)$, is expanded in $a_{s}$ keeping $\omega$ fixed,

$$
P^{\mathrm{FO}}\left(\omega, a_{s}\right)=\sum_{n=1}^{\infty} a_{s}^{n} P^{\mathrm{FO}(n-1)}(\omega),
$$

and truncated for some finite $n . P^{\mathrm{FO}}\left(\omega, a_{s}\right)$ can be obtained by subtracting all SGLs from the series for $P$ on the right hand side (RHS) of Eq. (12). Since all classes $m \leq 2 n$ are included in $P^{\mathrm{SGL}}$, i.e. since $P^{\mathrm{SGL}}$ contains all terms of the form $a_{s}^{n} / \omega^{p}$ for $p=0, \ldots, 2 n-1, P^{\mathrm{FO}}\left(\omega, a_{s}\right)$ is zero when $\omega=0$, since $P^{\mathrm{FO}(n)}(0)=0$. Fourthly and finally, the result for $P\left(\omega, a_{s}\right)$ is inverse Mellin transformed to obtain $P\left(x, a_{s}\right)$, and then Eq. (1) is solved exactly (which can be done numerically).

From (incomplete) calculations of Eq. (15) up to the class $m=2$ [6], $P$ is believed to be finite at $\omega=0$, and in particular to be a series in $\sqrt{a_{s}}$ with finite coefficients, beginning at $O\left(\sqrt{a_{s}}\right)$. This means that each term of the form $a_{s}^{n} / \omega^{p}$ for the types $p \geq 1$ in the expansion of Eq. (12) about $\omega=0$ should be included in the resummed term of class $m=2 n-p$. However, terms of the type $p=$ $0(m=2 n)$, which are included in our definition of SGLs, are non-singular and may therefore be left unresummed. Thus we define a second general scheme, which is the same as the SGL+FO scheme defined above, except that we separate $P^{\mathrm{SGL}}$ in Eq. (14) into

$$
P^{\mathrm{SGL}}=P_{p \geq 1}^{\mathrm{SGL}}+P_{p=0}^{\mathrm{SGL}},
$$

and expand only $P_{p \geq 1}^{\mathrm{SGL}}$ as in Eq. (15), while $P_{p=0}^{\mathrm{SGL}}$, which is independent of $\omega$, is expanded as a series in $a_{s}$. We shall call this the $\mathrm{SGL}+\mathrm{FO}+\mathrm{FO} \delta$ scheme, where "+FO $\delta$ " means that the $p=0$ terms, which are each proportional to $\delta(1-x)$ in $x$ space, are left as a FO series in $a_{s}$.

To summarize our SGL+FO $(+\mathrm{FO} \delta)$ scheme, we resum SGLs for which $m=1, \ldots, 2 n(m=1, \ldots, 2 n-1)$ in the form of Eq. (15), and treat all remaining terms as in the FO approach.

In the phase space region for $a_{s} \ll 1$ and $x$ above values for which $\ln (1 / x)=O\left(a_{s}^{-1 / 2}\right)$, the following $x$ space results indicate that the $\mathrm{SGL}+\mathrm{FO}(+\mathrm{FO} \delta)$ scheme gives a good description of the evolution. Firstly, $P_{p>1}^{\mathrm{SGL}}$, obtained from the inverse Mellin transform of Eq. (15) without terms of the type $p=0$, can be written as

$$
P_{p \geq 1}^{\mathrm{SGL}}\left(x, a_{s}\right)=\frac{1}{x \ln x} \sum_{m=1}^{\infty}\left(a_{s} \ln x\right)^{m} f_{m}\left(a_{s} \ln ^{2} x\right) .
$$

Equation (18) can also be obtained by summing the SGLs in $x$ space for each $m$. Since $a_{s} \ln x$ is always small, the series in Eq. (18) is a valid approximation when $x$ is small. On the other hand, as $x \rightarrow 1$ the SGLs for the types $p \geq 1$ all vanish, and therefore so does each term in the series in Eq. (18). Secondly, the full contribution from the type $p=0$ terms is just

$$
P_{p=0}^{\mathrm{SGL}}\left(x, a_{s}\right)=\delta(1-x) \sum_{n=1}^{\infty} C_{n} a_{s}^{n} .
$$

Thirdly and finally, the expansion of $P^{\mathrm{FO}}\left(x, a_{s}\right)$ in $a_{s}$, i.e. the inverse Mellin transform of Eq. (16),

$$
P^{\mathrm{FO}}\left(x, a_{s}\right)=\sum_{n=1}^{\infty} a_{s}^{n} P^{\mathrm{FO}(n-1)}(x),
$$

converges for all $x$.

\section{DLA IMPROVED DGLAP EVOLUTION}

In this section, we summarize the DLA and its relation to the DGLAP equation, and use this understanding to derive a modified form of DGLAP evolution in which all DLs, the $m=1$ class of SGLs, are included and resummed in a manner consistent with the 
$\mathrm{SGL}+\mathrm{FO}(+\mathrm{FO} \delta)$ scheme defined in Sec. III The DL contribution to the evolution obeys the DLA equation, which can be obtained from the DLA master equation for the quark and gluon generating functionals given in Ref. [6], and is given by

$$
\begin{array}{r}
\frac{d}{d \ln Q^{2}} D\left(x, Q^{2}\right)=\int_{x}^{1} \frac{d y}{y} \frac{2 C_{A}}{y} a_{s}\left(y^{2} Q^{2}\right) A D\left(\frac{x}{y}, y^{2} Q^{2}\right) \\
=\int_{x}^{1} \frac{d y}{y} \frac{2 C_{A}}{y} A y^{2 \frac{d}{d \ln Q^{2}}}\left[a_{s}\left(Q^{2}\right) D\left(\frac{x}{y}, Q^{2}\right)\right] .
\end{array}
$$

(The values of various factors are given in Appendix $\mathrm{A}$.) The result $y^{2 \frac{d}{d \ln Q^{2}}} f\left(Q^{2}\right)=f\left(y^{2} Q^{2}\right)$ has been used to obtain the second line in Eq. (21). Explicitly, $A=0$ for the DL evolving parts of the components $D=D_{q}^{-}$and $D=D_{N S}$, while

$$
A=\left(\begin{array}{cc}
0 & \frac{2 C_{F}}{C_{A}} \\
0 & 1
\end{array}\right)
$$

for the component $D=\left(D_{\Sigma}, D_{g}\right)$. Note that $A$ is a projection operator, i.e. it obeys

$$
A^{2}=A
$$

In Mellin space, Eq. (21), with certain boundary conditions to be discussed later, is equivalent to Eq. (11) with $P$ replaced by the $m=1$ term in Eq. (15),

$$
P^{\mathrm{DL}}\left(\omega, a_{s}\right)=\frac{a_{s}}{\omega} g_{1}\left(\frac{a_{s}}{\omega^{2}}\right)
$$

up to incomplete higher order terms in the remaining FO contribution and up to incomplete SGLs of classes for which $m \geq 2$.

The remaining part of the evolution (i.e. all the FO terms and remaining SGLs) can be included in Eq. (21) by writing it in the form

$$
\begin{gathered}
\frac{d}{d \ln Q^{2}} D\left(x, Q^{2}\right) \\
=\int_{x}^{1} \frac{d y}{y} \frac{2 C_{A}}{y} A y^{2 \frac{d}{d \ln Q^{2}}}\left[a_{s}\left(Q^{2}\right) D\left(\frac{x}{y}, Q^{2}\right)\right] \\
+\int_{x}^{1} \frac{d y}{y} \bar{P}\left(y, a_{s}\left(Q^{2}\right)\right) D\left(\frac{x}{y}, Q^{2}\right)
\end{gathered}
$$

$\bar{P}\left(x, a_{s}\right)$, which must be free of DLs, is constrained in terms of $P$ since Eq. 25) must be equivalent to Eq. (11) (for $D=D_{q}^{-}$and $D=D_{N S}$, one obtains the trivial result $\bar{P}=P$ ). In general, this can be done explicitly by expanding the operator in Eq. (25) in the form

$$
y^{2 \frac{d}{d \ln Q^{2}}}=\exp \left[2 \ln y \frac{d}{d \ln Q^{2}}\right]=\sum_{n=0}^{\infty} \frac{(2 \ln y)^{n}}{n !}\left(\frac{d}{d \ln Q^{2}}\right)^{n}
$$

and then repeatedly applying the evolution equations, Eqs. (11) and (2), to the $\left(\frac{d}{d \ln Q^{2}}\right)^{n}\left[a_{s}\left(Q^{2}\right) D\left(\frac{x}{y}, Q^{2}\right)\right]$ operations in Eq. (25). For example, to $O\left(a_{s}^{2}\right)$ one finds

$$
\begin{aligned}
\bar{P}\left(x, a_{s}\right)= & P\left(x, a_{s}\right)-2 C_{A} A\left[\frac{a_{s}}{x}+2 \beta\left(a_{s}\right) \frac{\ln x}{x}\right. \\
& \left.+\int_{x}^{1} \frac{d y}{y} \frac{2 a_{s} x \ln \frac{y}{x}}{y} P\left(y, a_{s}\right)\right]+O\left(a_{s}^{3}\right) .
\end{aligned}
$$

In the square brackets on the RHS of Eq. (27), only the first term contributes to the $O\left(a_{s}\right)(\mathrm{LO})$ part of $\bar{P}$, while the second and third term contribute to the $O\left(a_{s}^{2}\right)$ part. To this accuracy, the third term is calculated with $P\left(x, a_{s}\right)=a_{s} P^{(0)}(x)$, which can be found in the literature (see Appendix A). From Eq. (27) we observe that $\bar{P}$ up to $O\left(a_{s}^{2}\right)(\mathrm{NLO})$ is free of DLs, by taking the DLs in $P\left(x, a_{s}\right)$ for the first term on the RHS of Eq. (27) up to $O\left(a_{s}^{2}\right)$,

$$
P^{\mathrm{DL}}\left(x, a_{s}\right)=2 C_{A} \frac{A}{x} a_{s}-4 C_{A}^{2} \frac{A \ln ^{2} x}{x} a_{s}^{2}+O\left(a_{s}^{3}\right) .
$$

In practice, it is numerically very difficult to solve Eq. (25) (and Eq. (21)), in particular when $x<Q_{0} / Q$, which requires calculating the FFs for $Q<Q_{0}$. This problem corresponds simply to the fact that in addition to knowing $D\left(x, Q_{0}^{2}\right)$, which is required to solve Eq. (11), $\left.\frac{d}{d \ln Q^{2}} D\left(x, Q^{2}\right)\right|_{Q=Q_{0}}$ must also be known in order to solve Eq. (25). Even more seriously, Eq. (25) cannot be solved numerically at all when $x \leq \Lambda_{\mathrm{QCD}} / Q$, due to the Landau pole in $a_{s}\left(y^{2} Q^{2}\right)$.

Instead, we will examine what constraint Eq. (25) provides for $P$, since then the evolution can be performed using Eq. (1), which is numerically easily solved, without requiring explicit use of $D\left(x, Q^{2}\right)$ at scales less than $Q_{0}$ at any $x$. For this purpose, we work in Mellin space, where Eq. 25] becomes

$$
\begin{gathered}
\left(\omega+2 \frac{d}{d \ln Q^{2}}\right) \frac{d}{d \ln Q^{2}} D\left(\omega, Q^{2}\right)=2 C_{A} a_{s}\left(Q^{2}\right) A D\left(\omega, Q^{2}\right) \\
+\left(\omega+2 \frac{d}{d \ln Q^{2}}\right) \bar{P}\left(\omega, a_{s}\left(Q^{2}\right)\right) D\left(\omega, Q^{2}\right) .
\end{gathered}
$$

After substituting Eq. (6) into Eq. (29) and dividing out the overall factor of $D\left(\omega, Q^{2}\right)$, we obtain the following constraint on $P$ :

$$
\left(\omega+2 \frac{d}{d \ln Q^{2}}\right)(P-\bar{P})+2(P-\bar{P}) P-2 C_{A} a_{s} A=0 .
$$

Equations (25), (29) and (30) are exactly equivalent, however Eq. (30) shows most clearly that, for all $Q^{2}$, specifying $\bar{P}\left(\omega, a_{s}\left(Q^{2}\right)\right)$ will completely constrain $P\left(\omega, a_{s}\left(Q^{2}\right)\right)$ once $P\left(\omega, a_{s}\left(Q_{0}^{2}\right)\right)$ is chosen. However, the only information we have for $\bar{P}$ is that it is free of DLs, which means that an explicit constraint can be obtained 
only for $P^{\mathrm{DL}}$, which we now do. We first make the replacement

$$
P=\widetilde{P}+P^{\mathrm{DL}}
$$

in Eq. (30), where, comparing with Eq. (14),

$$
\widetilde{P}=P^{\mathrm{FO}}+P^{\mathrm{SGL}}-P^{\mathrm{DL}} \text {. }
$$

Then we expand Eq. (30) as a series in $a_{s} / \omega$ keeping $a_{s} / \omega^{2}$ fixed and extract the first, $O\left(\left(a_{s} / \omega\right)^{2}\right)$, term to find that the constraint on $P^{\mathrm{DL}}$ is exactly

$$
2\left(P^{\mathrm{DL}}\right)^{2}+\omega P^{\mathrm{DL}}-2 C_{A} a_{s} A=0 .
$$

Equation (33) gives two solutions for each component of $P$. Since $P$ is never larger than a $2 \times 2$ matrix in the basis consisting of singlet, gluon, non-singlet and valence quark FFs, there are four solutions which read

$$
\begin{aligned}
& P_{1, \pm}^{\mathrm{DL}}\left(\omega, a_{s}\right)=S_{ \pm}\left(\omega, a_{s}\right) A, \\
& P_{2, \pm}^{\mathrm{DL}}\left(\omega, a_{s}\right)=-\frac{\omega}{2} I-S_{ \pm}\left(\omega, a_{s}\right) A,
\end{aligned}
$$

where

$$
S_{ \pm}\left(\omega, a_{s}\right)=\frac{1}{4}\left(-\omega \pm \sqrt{\omega^{2}+16 C_{A} a_{s}}\right)
$$

For the evolution matrix $E$ defined in Eq. (8), these solutions for $P^{\mathrm{DL}}$ correspond respectively to

$$
\begin{aligned}
& E_{1, \pm}\left(\omega, a_{s}, a_{0}\right)=I+\left(e^{R_{ \pm}\left(\omega, a_{s}, a_{0}\right)}-1\right) A, \\
& E_{2, \pm}\left(\omega, a_{s}, a_{0}\right)=e^{P\left(\omega, a_{s}, a_{0}\right)} I \\
& \quad-e^{R_{\mp}\left(\omega, a_{s}, a_{0}\right)} \int_{a_{0}}^{a_{s}} d a \frac{S_{ \pm}(\omega, a)}{\beta(a)} e^{R_{ \pm}\left(\omega, a, a_{0}\right)} A,
\end{aligned}
$$

where we have used Eq. (23), and defined

$$
\begin{gathered}
R_{ \pm}\left(\omega, a_{s}, a_{0}\right)=\int_{a_{0}}^{a_{s}} d a \frac{S_{ \pm}(\omega, a)}{\beta(a)} \\
P\left(\omega, a_{s}, a_{0}\right)=-\frac{\omega}{2} \int_{a_{0}}^{a_{s}} \frac{d a}{\beta(a)} .
\end{gathered}
$$

Here, Eq. (2) has been used to transform the $\ln Q^{2}$ integrals into integrals over $a_{s}$. The general solution to the DL part of Eq. (29) is, finally, Eq. (8) with

$$
E\left(\omega, a_{s}, a_{0}\right)=\sum_{i, j} E_{i, j}\left(\omega, a_{s}, a_{0}\right) k_{i, j}\left(\omega, a_{0}\right)
$$

where $i=1,2$ and $j= \pm$. To ensure that $E$ is normalized as in the first line in Eq. (9), the matrices $k_{i, \pm}$ obey $\sum_{i, j} k_{i, j}\left(\omega, a_{0}\right)=I$. From Eq. (10), the most general splitting function is then

$$
\begin{aligned}
P^{\mathrm{DL}}\left(\omega, a_{s}\right)= & {\left[\sum_{i, j} P_{i, j}^{\mathrm{DL}}\left(\omega, a_{s}\right) E_{i, j}\left(\omega, a_{s}, a_{0}\right) k_{i, j}\left(\omega, a_{0}\right)\right] } \\
& \times\left[\sum_{i, j} E_{i, j}\left(\omega, a_{s}, a_{0}\right) k_{i, j}\left(\omega, a_{0}\right)\right]^{-1} .
\end{aligned}
$$

The only solution for $P^{\mathrm{DL}}\left(\omega, a_{s}\right)$ which is consistent with the DLs in the known result for $P$ in the FO approach of Eq. (11) to $O\left(a_{s}^{2}\right)$ (see Appendix A) is that for which $k_{1,+}=I$ and all the other $k_{i, j}$ are zero, i.e. $P^{\mathrm{DL}}=P_{1,+}^{\mathrm{DL}}$, or more explicitly,

$$
P^{\mathrm{DL}}\left(\omega, a_{s}\right)=\frac{A}{4}\left(-\omega+\sqrt{\omega^{2}+16 C_{A} a_{s}}\right),
$$

since, in the component $D=\left(D_{\Sigma}, D_{g}\right)$, the expansion of the result in Eq. (42) in $a_{s}$ to $O\left(a_{s}^{2}\right)$ keeping $\omega$ fixed gives

$$
P^{\mathrm{DL}}\left(\omega, a_{s}\right)=\left(\begin{array}{cc}
0 & a_{s} \frac{4 C_{F}}{\omega}-a_{s}^{2} \frac{16 C_{F} C_{A}}{\omega^{3}} \\
0 & a_{s} \frac{2 C_{A}}{\omega}-a_{s}^{2} \frac{8 C_{A}^{2}}{\omega^{3}}
\end{array}\right)+O\left(a_{s}^{3}\right),
$$

i.e. the Mellin transform of Eq. (28), while in the components $D=D_{q}^{-}$and $D=D_{N S}, P^{\mathrm{DL}}=0$. The other possibilities implied by Eq. (41) do not give these results and/or cannot be expanded in $a_{s}$, i.e. they contain non-perturbative terms. Equation (42) agrees with the results of Ref. [8], which are derived using the conventional renormalization group approach, and with the results from the generating functional technique of Ref. 6]. Thus, the explicit evolution is given by Eq. (36) with the upper sign. Writing $R_{+}=R$ and returning to Eq. (8), the evolution obeys

$$
D\left(\omega, Q^{2}\right)=\left[I+\left(e^{R\left(\omega, a_{s}\left(Q^{2}\right), a_{s}\left(Q_{0}^{2}\right)\right)}-1\right) A\right] D\left(\omega, Q_{0}^{2}\right) .
$$

For all DLs in the evolution, Eq. (44) solves Eq. (21).

A completely explicit form for Eq. (44) can be obtained in the case that Eq. (3) is taken to $O\left(a_{s}^{2}\right)$ only. Equation (2) then implies that $a_{s}\left(Q^{2}\right)=1 /\left(\beta_{0} \ln \left(Q^{2} / \Lambda_{\mathrm{QCD}}^{2}\right)\right)$, for which Eq. (38) (with the upper sign) reads

$$
\begin{aligned}
& R\left(\omega, a_{s}, a_{0}\right)=\frac{1}{4 \beta_{0} a_{s}}\left(-\omega+\sqrt{\omega^{2}+16 C_{A} a_{s}}\right) \\
& +\frac{2 C_{A}}{\omega \beta_{0}} \ln \frac{\omega+\sqrt{\omega^{2}+16 C_{A} a_{s}}}{-\omega+\sqrt{\omega^{2}+16 C_{A} a_{s}}}-\left(a_{s} \leftrightarrow a_{0}\right) .
\end{aligned}
$$

Since $P_{\Sigma \Sigma}^{\mathrm{DL}}=P_{g \Sigma}^{\mathrm{DL}}=0$ according to Eq. (42), it follows from Eq. (6) when $P=P^{\mathrm{DL}}$ that, for small $\omega$,

$$
\frac{d}{d \ln Q^{2}} D_{\Sigma}\left(\omega, Q^{2}\right)=\frac{P_{\Sigma g}^{\mathrm{DL}}\left(\omega, a_{s}\left(Q^{2}\right)\right)}{P_{g g}^{\mathrm{DL}}\left(\omega, a_{s}\left(Q^{2}\right)\right)} \frac{d}{d \ln Q^{2}} D_{g}\left(\omega, Q^{2}\right) \text {. }
$$


With the results for $P_{\Sigma g}^{\mathrm{DL}}$ and $P_{g g}^{\mathrm{DL}}$ in Eq. (42), integrating Eq. (46) over $\ln Q^{2}$ gives

$$
D_{\Sigma}=\frac{2 C_{F}}{C_{A}} D_{g}
$$

The constant of integration has been neglected in Eq. (47), which is valid for large $Q$. Equation (47) (again up to an additional constant) can also be derived from Eq. (44).

Since the non-singlet and valence quark splitting functions are free of DLs, the derivatives of the non-singlet and valence quark FFs with respect to $\ln Q^{2}$ may be neglected at small $\omega$. Again, integrating such results over $\ln Q^{2}$ and neglecting the constants of integration implies that the non-singlet and valence quark FFs vanish. In this case Eq. (47) becomes

$$
D_{q, \bar{q}}=\frac{C_{F}}{C_{A}} D_{g}
$$

reducing the number of FFs required for the cross section to one, $D_{g}$. Such a low $x$ approximation is often used in DLA or MLLA analyses of data. However, since we want a complete formalism suitable for both small and large $x$, we will only use Eq. (48) to partially constrain our choice of parameterization at low $x$ in the next section.

Complete information on the SL contribution to $P^{\mathrm{SGL}}$, given in the notation in Eq. (15) by

$$
P^{\mathrm{SL}}\left(\omega, a_{s}\right)=\left(\frac{a_{s}}{\omega}\right)^{2} g_{2}\left(\frac{a_{s}}{\omega^{2}}\right),
$$

cannot be obtained from Eq. (30), since the full SL contribution to $\bar{P}$ is not known. However, its SL at $O\left(a_{s}\right)$, which according to Eq. (27) is equal to the SL in $P$ at $O\left(a_{s}\right)$, given according to Appendix $\mathrm{A}$ by

$$
P^{\mathrm{SL}(0)}(\omega)=\left(\begin{array}{cc}
0 & -3 C_{F} \\
\frac{2}{3} T_{R} n_{f} & -\frac{11}{6} C_{A}-\frac{2}{3} T_{R} n_{f}
\end{array}\right),
$$

is a type $p=0$ term (see Sec. (II). Therefore, in Eq. (30), approximating $\bar{P}$ by $a_{s} P^{\mathrm{SL}(0)}$ should lead to a better approximation for the evolution than approximating $\bar{P}$ by zero. Not surprisingly, with this approximation, Eq. (29) can be regarded as a generalized version of the MLLA equation to include quarks, in the sense that the $g$ component of this latter equation for $D=\left(D_{\Sigma}, D_{g}\right)$ when Eq. (47) is invoked is precisely the MLLA equation of Ref. [6]. We therefore conclude that although Eq. (29) is derived from the DLA, it is more complete than the MLLA equation since in Eq. (29) it is neither necessary to restrict $\bar{P}$ in this way nor to use the approximation in Eq. (47).

We may now approximately but explicitly calculate the evolution in the $\mathrm{SGL}+\mathrm{FO}(+\mathrm{FO} \delta)$ scheme by approximating $P^{\mathrm{SGL}}$ in Eq. (14) by its leading term $P^{\mathrm{DL}}$ given by Eq. (42), being the first, $O\left(a_{s} / \omega\right)$, term in Eq. (15). Thus we take

$$
P=P^{\mathrm{DL}}+P^{\mathrm{FO}}\left(+P_{p=0}^{\mathrm{SGL}}\right)
$$

Recall that $P^{\mathrm{FO}}$ is equal to $P$ in Eq. (12) when all SGLs are excluded. After inverse Mellin transforming Eq. (51), we can solve Eq. (10) directly using standard numerical techniques for $x$ space evolution, as suggested in Sec. III We shall call this the $\mathrm{DL}+\mathrm{FO}(+\mathrm{FO} \delta)$ scheme. We note that, although the analytic solution to Eq. (6) for $P=P^{\mathrm{DL}}$ as defined in Eq. (42) is given by Eq. (44), while the analytic solution to Eq. (6) for $P=P^{\mathrm{FO}}$ can be found using the well known method in the FO approach, an analytic solution to Eq. (6) for $P$ given in Eq. (51) does not seem feasible.

For the $\mathrm{DL}+\mathrm{FO}(+\mathrm{FO} \delta)$ scheme, we require $P^{\mathrm{DL}}\left(x, a_{s}\right)$. The inverse Mellin transform of Eq. (42) gives

$$
P^{\mathrm{DL}}\left(x, a_{s}\right)=\frac{A \sqrt{C_{A} a_{s}}}{x \ln \frac{1}{x}} J_{1}\left(4 \sqrt{C_{A} a_{s}} \ln \frac{1}{x}\right),
$$

where $J_{1}(y)$ is the Bessel function of the first kind, given by

$$
J_{1}(y)=\frac{y}{2} \sum_{r=0}^{\infty} \frac{\left(\frac{-y^{2}}{4}\right)^{r}}{r !(r+1) !}
$$

This gives

$$
P^{\mathrm{DL}}\left(x, a_{s}\right)=\frac{2 C_{A} a_{s} A}{x} \sum_{r=0}^{\infty} \frac{(-1)^{r}}{r !(r+1) !}\left(4 C_{A} a_{s} \ln ^{2} x\right)^{r} .
$$

The series in Eq. (54) may also be obtained by expanding Eq. (42) to infinite order in $a_{s}$, using Eq. (13) to perform the inverse Mellin transform on each term, and finally using the identity

$$
(-4)^{r} \frac{\left(-\frac{1}{2}\right)\left(-\frac{3}{2}\right) \cdots\left(\frac{3}{2}-r\right)\left(\frac{1}{2}-r\right)}{(2 r) !}=\frac{1}{r !}
$$

for $r \geq 0$. Equation (54) in fact converges rapidly, however the truncated series can differ substantially from the full series whenever $x$ is small enough. For more reliability, the Bessel function should be calculated by numerical evaluation of the result

$$
J_{1}(y)=\frac{1}{\pi} \int_{0}^{\pi} d \theta \cos (y \sin \theta-\theta)
$$

Note that for $Q>\Lambda_{\mathrm{QCD}}$, the evolution of $D\left(x, Q^{2}\right)$ in the $\mathrm{DL}+\mathrm{FO}(+\mathrm{FO} \delta)$ scheme contains no Landau pole for all $x$, not just for $x>\Lambda_{\mathrm{QCD}} / Q$ as is the case if we evolve with Eq. (25).

Using the property at large $y$ that

$$
J_{1}(y)=\sqrt{\frac{2}{\pi y}} \cos \left(y-\frac{3 \pi}{4}\right)+O\left(\frac{1}{y}\right),
$$


we find the true $x \rightarrow 0$ divergence

$$
\begin{aligned}
P^{\mathrm{DL}}\left(x, a_{s}\right) & =\frac{\left(C_{A} a_{s}\right)^{\frac{1}{4}} A}{\sqrt{2 \pi} x \ln ^{\frac{3}{2}} \frac{1}{x}} \cos \left(4 \sqrt{C_{A} a_{s}} \ln \frac{1}{x}-\frac{3 \pi}{4}\right) \\
& +O\left(\frac{1}{x \ln ^{2} \frac{1}{x}}\right)
\end{aligned}
$$

of $P$ to be weaker than the one from the FO approach, which from Eq. (54) is proportional to $(1 / x) \ln ^{2(n-1)}(1 / x)$ at $O\left(a_{s}^{n}\right)$.

For simplicity, in the next section we shall take $P^{\mathrm{FO}}$ (and $\left.P_{p=0}^{\mathrm{SGL}}\right)$ to $O\left(a_{s}\right)$ only in the $\mathrm{DL}+\mathrm{FO}(+\mathrm{FO} \delta)$ scheme. In other words, we will approximate $P$ by

$$
P=P^{\mathrm{DL}}+a_{s} P^{\mathrm{FO}(0)}\left(+a_{s} P^{\mathrm{SL}(0)}\right) .
$$

We shall call this the $\mathrm{DL}+\mathrm{LO}(+\mathrm{LO} \delta)$ scheme.

It is well known from the MLLA that while DLs give the shape of the peak that occurs at small $x$, the gluon component of the type $p=0 \mathrm{SL}$ is required to get the correct peak position. We therefore anticipate that, relative to both the DL+LO scheme and the MLLA, the $\mathrm{DL}+\mathrm{LO}+\mathrm{LO} \delta$ scheme will give a better description of the data since it contains both gluon and quark components of the type $p=0 \mathrm{SL}$.

In Fig. 1] we see that $P_{g g}\left(x, a_{s}\right)$ in the DL+LO scheme, which is equal to the $\mathrm{DL}+\mathrm{LO}+\mathrm{LO} \delta$ scheme when $x \neq 1$, interpolates well between its $O\left(a_{s}\right)$ approximation in the FO approach at large $x$ and $P_{g g}^{\mathrm{DL}}\left(x, a_{s}\right)$ at small $x$ (the small difference here comes from $P^{\mathrm{FO}(0)}(x)$ at small $x$ ). DL resummation clearly makes a large difference to $P$ at small $x$.

\section{COMPARISONS WITH DATA}

In this section, we elaborate on our numerical study of the $\mathrm{DL}+\mathrm{LO}+\mathrm{LO} \delta$ scheme in Ref. 10]. We perform a numerical comparison of the $\mathrm{FO}$ and $\mathrm{DL}+\mathrm{LO}+\mathrm{LO} \delta$ schemes by otherwise imposing the same assumptions and choice of parameterization, starting scale etc., and then fitting in each scheme to precisely the same experimental measurements of the normalised differential cross section for light charged hadron production in the process $e^{+} e^{-} \rightarrow(\gamma, Z) \rightarrow h+X$, where $h$ is the observed hadron and $X$ is anything else. This data, spread over a wide range in center-of-mass energy $\sqrt{s}$, is composed of the sets from TASSO at $\sqrt{s}=14,35,44 \mathrm{GeV}$ [13] and $22 \mathrm{GeV}$ [14], MARK II [15] and TPC [16] at $29 \mathrm{GeV}$, TOPAZ at $58 \mathrm{GeV}$ [17], ALEPH [18], DELPHI 19], L3 20], OPAL 21] and SLC 22] at 91 GeV, ALEPH 23. and OPAL 24 at $133 \mathrm{GeV}$, DELPHI at $161 \mathrm{GeV} 25$ and OPAL at 172, 183, $189 \mathrm{GeV}[26]$ and $202 \mathrm{GeV}$ [27]. These data span a wide range in $x_{p}=2 p / \sqrt{s}$, where $p$ is the momentum of the observed hadron, which constrain

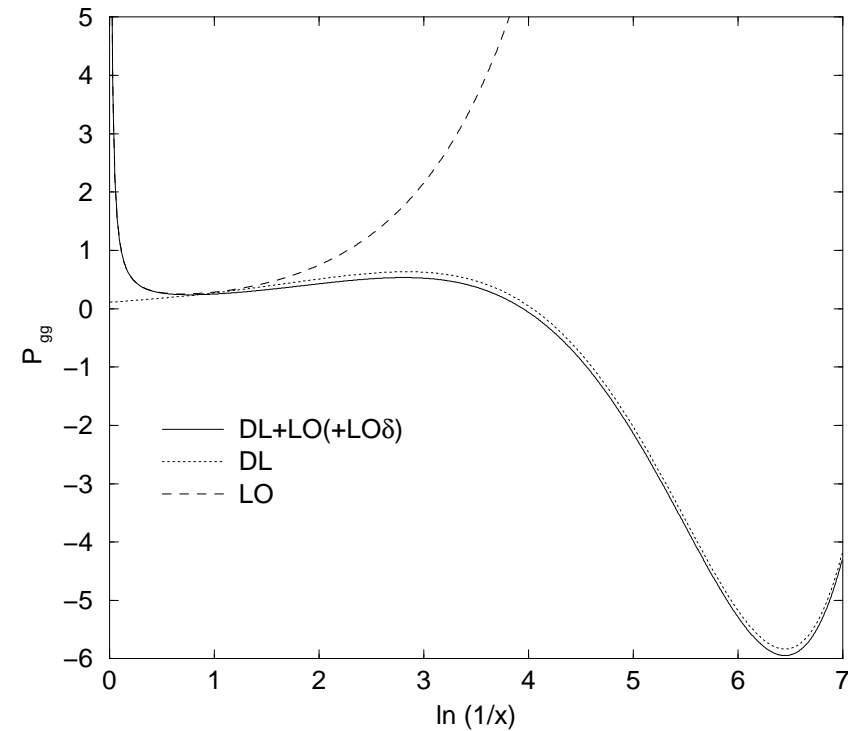

FIG. 1: (i) $P_{g g}\left(x, a_{s}\right)$ calculated in the $\mathrm{DL}+\mathrm{LO}(+\mathrm{LO} \delta)$ scheme, (ii) $P_{g g}\left(x, a_{s}\right)$ calculated to $O\left(a_{s}\right)$ in the FO approach (labelled "LO"), and (iii) $P_{g g}^{\mathrm{DL}}\left(x, a_{s}\right)$ (labelled "DL"). $a_{s}=0.118 /(2 \pi)$.

the FFs in the region of $x$ for which $x_{p} \leq x \leq 1$. In Ref. [12], large $\xi$ data could be described within the range

$$
\xi<\ln \frac{\sqrt{s}}{2 M}
$$

where $\xi=\ln \left(1 / x_{p}\right)$ and $M$ is a mass scale of $O(1) \mathrm{GeV}$. In Ref. [9], this inability to describe large $\xi$ (small $x_{p}$ ) data using the naive approach to the MLLA was attributed to the formally beyond-MLLA evolutions of the higher moments (skewness, kurtosis etc.) becoming too large at large $\sqrt{s}$, and when these were fixed to be zero it was found that the MLLA could give an excellent description of data up to the highest values of $\xi$ currently measured. In the $\mathrm{DL}+\mathrm{LO}+\mathrm{LO} \delta$ scheme here, the evolution of the higher moments should be somewhat suppressed by the FO contribution [9]. However, we will nevertheless impose Eq. (60) on the data to be fitted to, since here our aim is to extend the good FO DGLAP description of small $\xi$ (large $x_{p}$ ) data to larger $\xi$ via DL resummation.

At LO in the coefficient functions, these data are described in terms of the evolved FFs by

$$
\frac{1}{\sigma(s)} \frac{d \sigma}{d x_{p}}\left(x_{p}, s\right)=\frac{1}{n_{f}\langle Q(s)\rangle} \sum_{q} Q_{q}(s) D_{q}^{+}\left(x_{p}, Q^{2}\right),
$$

where $Q_{q}$ is the electroweak charge of a quark with flavour $q$ and $\langle Q\rangle$ is the average charge over all flavours. For Eq. (61) to be a valid approximation, it is necessary to choose $Q=O(\sqrt{s})$. Since we only use data for which $\sqrt{s}>m_{b}$, where $m_{b} \approx 5 \mathrm{GeV}$ is the mass of the bottom quark, and since later we will also set $Q_{0}>m_{b}$, we will take $n_{f}=5$ in all our calculations. While the 
precise choice for $n_{f}$ does not matter in the DLA, calculations in the FO approach depend strongly on it. Since we sum over hadron charges, we set $D_{\bar{q}}=D_{q}$. Since we do not use data with quark tagging, the $c$ quark cannot be distinguished from the $u$ quark since both quarks couple to the $Z$ boson in the same way, i.e. have the same electroweak charge. Likewise, the $d, s$ and $b$ quarks are similar to one another in this respect. Therefore, to avoid redundant degrees of freedom, we fit only the FFs

$$
\begin{aligned}
f_{u c}\left(x, Q_{0}^{2}\right) & =\frac{1}{2}\left(u\left(x, Q_{0}^{2}\right)+c\left(x, Q_{0}^{2}\right)\right) \\
f_{d s b}\left(x, Q_{0}^{2}\right) & =\frac{1}{3}\left(d\left(x, Q_{0}^{2}\right)+s\left(x, Q_{0}^{2}\right)+b\left(x, Q_{0}^{2}\right)\right)
\end{aligned}
$$

and the gluon $g\left(x, Q_{0}^{2}\right)$. For each of these three FFs, we choose the parameterization

$$
f\left(x, Q_{0}^{2}\right)=N \exp \left[-c \ln ^{2} x\right] x^{\alpha}(1-x)^{\beta},
$$

since at intermediate and large $x$ the $\mathrm{FF}$ is constrained to behave like

$$
f\left(x, Q_{0}^{2}\right) \approx N x^{\alpha}(1-x)^{\beta},
$$

which is the standard parameterization used in global fits at large $x$, while at small $x$ (where $\left.(1-x)^{\beta} \approx 1\right)$ the FF is constrained to behave like

$$
\lim _{x \rightarrow 0} f\left(x, Q_{0}^{2}\right)=N \exp \left[-c \ln ^{2} \frac{1}{x}-\alpha \ln \frac{1}{x}\right],
$$

which for $c>0$ is a Gaussian in $\ln (1 / x)$ of width $1 / \sqrt{2 c}$, center at $-\alpha /(2 c)$ and normalization given by $N \sqrt{\pi / c} \exp \left[\alpha^{2} /(4 c)\right]$. For sufficiently large $Q_{0}$, the DLA predicts such behaviour with $\alpha<0$.

In addition, we use Eq. (48) to remove four free parameters by imposing the constraints

$$
\begin{aligned}
& c_{u c}=c_{d s b}=c_{g}, \\
& \alpha_{u c}=\alpha_{d s b}=\alpha_{g} .
\end{aligned}
$$

This implies that all FFs have the same width and center, however the normalisations may not turn out to be consistent with Eq. (48). However, Eq. (48) is only an approximation at small $x$, while the $N$ are also relevant in the large $x$ region, where also the $(1-x)^{\beta}$ factors are necessary. At any rate, it will be interesting to see how well the relation

$$
N_{u c} \approx N_{d s b} \approx \frac{C_{F}}{C_{A}} N_{g}
$$

as implied by Eq. (48) is obeyed after a fit is performed. In addition to the 8 free parameters for the FFs, we also fit $\Lambda_{\mathrm{QCD}}$. We choose $Q^{2}=s$, although it is only important that the latter two quantities are kept proportional, since the constant of proportionality has no effect on the final FF parameters and the description of the data (or, equivalently, the quality of the fit). However, the final fitted $\Lambda_{\mathrm{QCD}}$ varies in proportion to this constant, so there will be an overall theoretical error on our fitted values for $\Lambda_{\mathrm{QCD}}$ of a factor of $O(1)$. Since all data will be at $\sqrt{s} \geq 14 \mathrm{GeV}$, we choose $Q_{0}=14 \mathrm{GeV}$. As discussed in Sec. III the evolution is performed by numerically integrating Eq. (11). For this we use a grid consisting of 250 points equally spaced in $\ln Q^{2}$ over the range 14 $\mathrm{GeV} \leq Q \leq 202 \mathrm{GeV}$, and 750 points equally spaced in $\ln (1 / x)$ over the range $0 \leq \ln (1 / x) \leq 11.6$.

\section{A. Fixed Order Evolution}

We first perform a fit to all data sets listed above using DGLAP evolution in the FO approach to LO, without DL resummation. This approach is the same as that used in fits in the literature. We fit to those data for which Eq. (60) is obeyed with $M=0.5 \mathrm{GeV}$. This gives a total of 425 data points out of the available 492 . We obtain a $\chi^{2}$ per degree of freedom $\chi_{\mathrm{DF}}^{2}=3.0$ (or 2.1 after subtraction of the contribution to $\chi^{2}$ from the TOPAZ data, which is the only data set from which an individual $\chi_{\mathrm{DF}}^{2}$ greater than 6 is obtained), and the results are shown in Fig. 2 and Table I] The result for $\Lambda_{\mathrm{QCD}}$ is quite consistent with that of other analyses, at least within the theoretical error. It is clear that FO DGLAP evolution fails in the description of the peak region and shows a different trend outside the fit range. The $\exp \left[-c \ln ^{2} x\right]$ factor does at least allow for the fit range to be extended to $x$ values below that of $x=0.1$, the lower limit of most global fits, to around $x=0.05(\xi=3)$ for data at the larger $\sqrt{s}$ values. Note that the negative value of $\beta$ for the gluon is

\begin{tabular}{|c|c|c|c|c|}
\hline $\mathrm{FF} \quad$ Parameter & $N$ & $\beta$ & $\alpha$ & $c$ \\
\hline$g$ & 0.22 & -0.43 & -2.38 & 0.25 \\
\hline$u+c$ & 0.49 & 2.30 & {$[-2.38]$} & {$[0.25]$} \\
\hline$d+s+b$ & 0.37 & 1.49 & {$[-2.38]$} & {$[0.25]$} \\
\hline
\end{tabular}
unphysical, because the gluon FF is weakly constrained in our fit since it couples to the data only through the evolution (see Eq. (61)).

TABLE I: Parameter values for the FFs at $Q_{0}=14 \mathrm{GeV}$ parameterized as in Eq. (63) from a fit to all data listed in the text using DGLAP evolution in the FO approach to LO. $\Lambda_{\mathrm{QCD}}=388 \mathrm{MeV}$.

\section{B. Incorporation of Soft Gluon Resummation}

We now perform the same fit, i.e. to the same data with the same parameterization, but now evolving in the $\mathrm{DL}+\mathrm{LO}+\mathrm{LO} \delta$ scheme. The results are shown in Table III and Fig. [3. We obtain $\chi_{\mathrm{DF}}^{2}=2.1$ (or 1.4 without the TOPAZ data - for each remaining data set, the individual $\chi_{\mathrm{DF}}^{2}$ is less than 3), a significant improvement to the fit above with FO DGLAP evolution. The data 


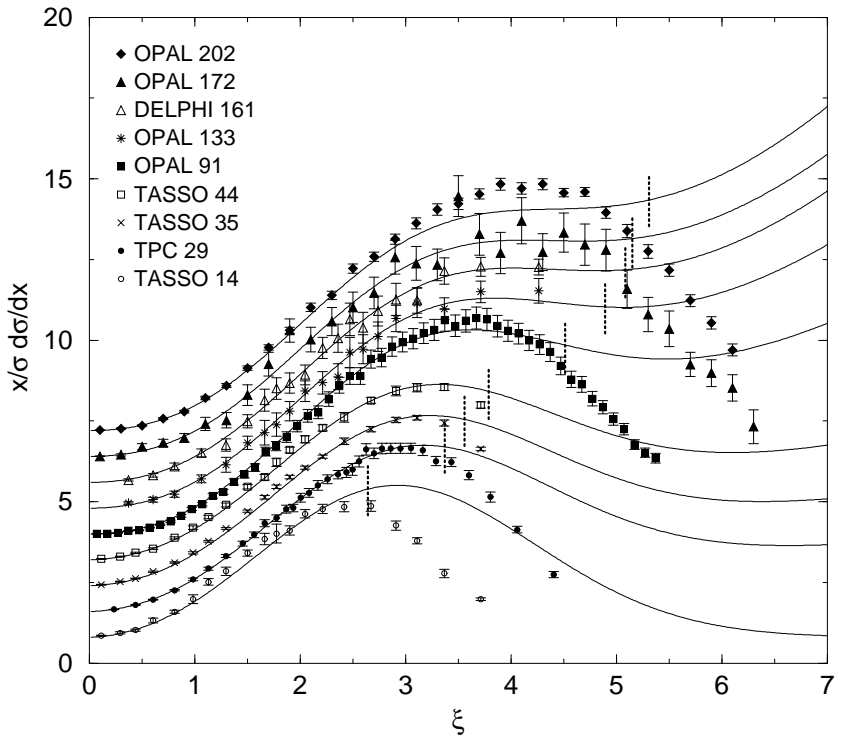

FIG. 2: Fit to data as described in Table 1 Some of the data sets used for the fit are shown, together with their theoretical predictions from the results of the fit. Data to the right of the horizontal dotted lines have not been used in the fit. Each curve is shifted up by 0.8 for clarity.

TABLE II: Parameter values for the FFs at $Q_{0}=14 \mathrm{GeV}$ parameterized as in Eq. 63) from a fit to all data listed in the text using DGLAP evolution in the $\mathrm{DL}+\mathrm{LO}+\mathrm{LO} \delta$ scheme. $\Lambda_{\mathrm{QCD}}=801 \mathrm{MeV}$.

\begin{tabular}{|c|c|c|c|c|}
\hline $\begin{array}{ll}\mathrm{FF} & \text { Parameter } \\
\end{array}$ & $N$ & $\beta$ & $\alpha$ & $c$ \\
\hline$g$ & 1.60 & 5.01 & -2.63 & 0.35 \\
\hline$u+c$ & 0.39 & 1.46 & {$[-2.63]$} & {$[0.35]$} \\
\hline$d+s+b$ & 0.34 & 1.49 & {$[-2.63]$} & {$[0.35]$} \\
\hline
\end{tabular}

around the peak is now much better described. The energy dependence is well reproduced up to the largest $\sqrt{s}$ value, $\sqrt{s}=202 \mathrm{GeV}$. At $\sqrt{s}=14 \mathrm{GeV}$, the low $x$ description of the data is extended from $x=0.1$ in the unresummed case down to 0.06 in the resummed case, and from $x=0.05$ to 0.005 at $\sqrt{s}=202 \mathrm{GeV}$. This should also be compared to the fit to the same data in Ref. 12. where DL resummation was used within the MLLA but with neither FO terms nor quark freedom (i.e. Eq. (48) was imposed over the whole $x$ range). That fit gave $\chi_{\mathrm{DF}}^{2}=4.0$. We conclude that, relative to the MLLA, the FO contributions in the evolution, together with freedom from the constraint of Eq. (48), makes a significant improvement to the description of the data for $\xi$ from zero to just beyond the peak.

The value $\Lambda_{\mathrm{QCD}} \approx 800 \mathrm{MeV}$ is somewhat larger than the value $480 \mathrm{MeV}$ which we obtain from a DGLAP fit in the large $x$ range $(x>0.1)$. We note that had we made the usual DLA (MLLA) choice $Q=\sqrt{s} / 2$ instead of our choice $Q=\sqrt{s}$ which is usually employed in analyses using the DGLAP equation, we would have obtained half

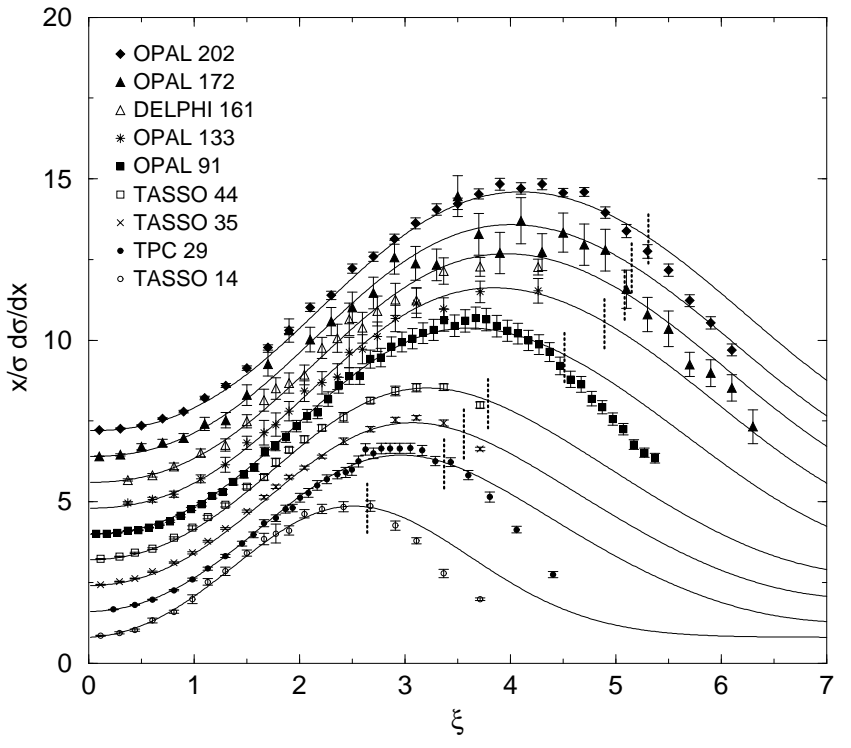

FIG. 3: Fit to data as described in Table III

this value for $\Lambda_{\mathrm{QCD}} \cdot N_{g}$ is too large by a factor of about 2 relative to its prediction in Eq. (67). As noted before, the initial gluon FF is weakly constrained in our fits since it only enters the cross section via the mixing with the quark FFs in the evolution.

While the DL resummation greatly improves the description around the peak, the description still deteriorates as $\xi$ increases, since a fit with data at larger $\xi$ resulted in an increase in $\Lambda_{\mathrm{QCD}}$ and $N_{g}$, as well as $\chi_{\mathrm{DF}}^{2}$. The large $\xi$ data may be better described with the inclusion of the (unknown) resummed SL contribution. Indeed, fitting without the LO $p=0$ term (the LO SL), i.e. fitting in the $\mathrm{DL}+\mathrm{LO}$ scheme, generally gives a larger $\chi_{\mathrm{DF}}^{2}$ compared to the same fits in the $\mathrm{DL}+\mathrm{LO}+\mathrm{LO} \delta$ scheme, since the data around the peak region could not be described.

We repeated the above two fits without the constraints given in Eq. (66) and found no substantial improvement to the fits.

The results of these two fits show that, for the DGLAP evolution to describe the overall features of the data from small to large $x_{p}$, resummation of SGLs is necessary. In addition, our DL resummation scheme proves to be an adequate implementation of this resummation. However, the description at $\xi$ values beyond the peak remain inaccessible. It is clear from the smoothness of the shape around and beyond the peak in Fig. 3] that this cannot be attributed to any instability in the evolution of the higher moments. This instability occurred in the method of Ref. 12], which was pointed out and remedied in Ref. [9]. Figure 3 in fact shows that the evolution at large $\xi$ follows the data well. Indeed, a fit in the $\mathrm{DL}+\mathrm{LO}+\mathrm{LO} \delta$ scheme to data in the same region as that used in the fits of Ref. 9],

$$
\xi>0.75+0.33 \ln (\sqrt{s}),
$$


gives $\chi_{\mathrm{DF}}^{2}=7$, which is admittedly unacceptably large, however an FO fit to the same data gives $\chi_{\mathrm{DF}}^{2}=35$. Thus there is a problem with simultaneously describing both the range from $\xi=0$ to the peak region to the range beyond the peak within the $\mathrm{DL}+\mathrm{LO}+\mathrm{LO} \delta$ scheme, implying a large theoretical error beyond the peak. This may be due to the neglect of the complete SL contribution, which is unfortunately unknown. Another likely reason is our neglect of the effect of the produced hadron's mass. This effect is important at small $x_{p}$. We will therefore study this effect in the next subsection.

\section{Incorporation of Hadron Mass Effects}

We will now incorporate hadron mass effects into our calculations, using a specific choice of scaling variable. An alternative approach is given in Ref. 28] (see also Ref. 29]). For this purpose it is helpful to work with light cone coordinates, in which any 4-vector $V$ is written in the form $V=\left(V^{+}, V^{-}, \mathbf{V}_{T}\right)$ with $V^{ \pm}=\frac{1}{\sqrt{2}}\left(V^{0} \pm V^{3}\right)$ and $\mathbf{V}_{T}=\left(V^{1}, V^{2}\right)$. In the center-of-mass (COM) frame, the momentum of the electroweak boson takes the form

$$
q=\left(\frac{\sqrt{s}}{\sqrt{2}}, \frac{\sqrt{s}}{\sqrt{2}}, \mathbf{0}\right) .
$$

In the absence of hadron mass, $x_{p}$ (whose definition $x_{p}=2 p / \sqrt{s}$ applies only in the COM frame) is identical to the light cone scaling variable $\eta=p_{h}^{+} / q^{+}$. However, the definition $x_{p}=2 p / \sqrt{s}$ applies only in the COM frame, so $\eta$ is a more convenient scaling variable for studying hadron mass effects since it is invariant with respect to boosts along the direction of the hadron's spatial momentum. Taking this direction to be the 3 -axis, and introducing a mass $m_{h}$ for the hadron, the momentum of the hadron in the COM frame reads

$$
p_{h}=\left(\frac{\eta \sqrt{s}}{\sqrt{2}}, \frac{m_{h}^{2}}{\sqrt{2} \eta \sqrt{s}}, \mathbf{0}\right) \text {. }
$$

Therefore the relation between the two scaling variables in the presence of hadron mass is

$$
x_{p}=\eta\left(1-\frac{m_{h}^{2}}{s \eta^{2}}\right) .
$$

Note that these two variables are approximately equal when $m_{h} \ll x_{p} \sqrt{s}$, i.e. hadron mass effects cannot be neglected when $x_{p}$ (or $\eta$ ) are too small.

In the leading twist component of the cross section after factorization, the hadron $h$ is produced by fragmentation from a real, massless parton of momentum

$$
k=\left(\frac{p_{h}^{+}}{y}, 0, \mathbf{0}\right) .
$$

The + component of everything other than this parton and of everything produced by the parton other than the observed hadron $h$ must be positive, implying $y \geq$ $\eta$ and $y \leq 1$ respectively. As a generalization of the massless case, we assume the cross section we have been calculating is $(d \sigma / d \eta)(\eta, s)$, i.e.

$$
\frac{d \sigma}{d \eta}(\eta, s)=\int_{\eta}^{1} \frac{d y}{y} \frac{d \sigma}{d y}\left(y, s, Q^{2}\right) D\left(\frac{\eta}{y}, Q^{2}\right)
$$

which is related to the measured observable $\left(d \sigma / d x_{p}\right)\left(x_{p}, s\right)$ via

$$
\frac{d \sigma}{d x_{p}}\left(x_{p}, s\right)=\frac{1}{1+\frac{m_{h}^{2}}{s \eta^{2}\left(x_{p}\right)}} \frac{d \sigma}{d \eta}\left(\eta\left(x_{p}\right), s\right) .
$$

Note that the effect of hadron mass is to reduce the size of the cross section at small $x_{p}$ (or $\eta$ ), which Fig. 3 suggests is what is needed to improve the fit. The problem with the above method is that it only applies to the case where only one species of hadron is produced, whereas the data we are studying are for light charged hadrons, i.e. charged pions, charged kaons and the (anti)protons, whose masses (140, 494 and $938 \mathrm{MeV}$, respectively) are substantially different. However, since most of the produced particles will be pions and kaons, the effective range of hadron masses may be sufficiently small to achieve reliable results when the particle masses are taken to be equal, which we will do. We now perform the last two fits again but with $m_{h}$ included in the list of free parameters. For FO evolution, we obtain $\chi_{\mathrm{DF}}^{2}=2.06$, which is a substantial improvement over the same fit above for which no treatment of hadron mass effects is applied. The results are shown in Fig. 4 and Table III The result for $m_{h}$ is of the expected order of magnitude, however $\Lambda_{\mathrm{QCD}}$ is unreasonably large. The

\begin{tabular}{|c|c|c|c|c|}
\hline $\mathrm{FF} \quad$ Parameter & $N$ & $\beta$ & $\alpha$ & $c$ \\
\hline$g$ & 0.11 & -0.82 & -2.01 & 0.18 \\
\hline$u+c$ & 0.70 & 2.12 & {$[-2.01]$} & {$[0.18]$} \\
\hline$d+s+b$ & 0.82 & 2.35 & {$[-2.01]$} & {$[0.18]$} \\
\hline
\end{tabular}
suppression of the cross section beyond the peak from hadron mass effects is evident, and allows for the cross section to follow the data much more closely.

TABLE III: As in Table $\llbracket$ but incorporating mass effects in the fit. $\Lambda_{\mathrm{QCD}}=1308 \mathrm{MeV}$ and $m_{h}=408 \mathrm{MeV}$.

For the $\mathrm{DL}+\mathrm{LO}+\mathrm{LO} \delta$ fit, we obtain the results in Fig. [5] and Table IV] The parameters are not substantially different to those in Table The result for $m_{h}$ is again reasonable. We find $\chi_{\mathrm{DF}}^{2}=2.03$, i.e. the quality of the fit is the same as for the previous FO fit, showing that the FO case benefits much more from the inclusion of mass effects than the DL resummed case. However, treatment of mass effects renders the value of $\Lambda_{\mathrm{QCD}}$ obtained in the fit with DL resummation more reasonable.

We conclude therefore that to improve the large $\xi$ description and to achieve a reasonable value for $\Lambda_{\mathrm{QCD}}$, 


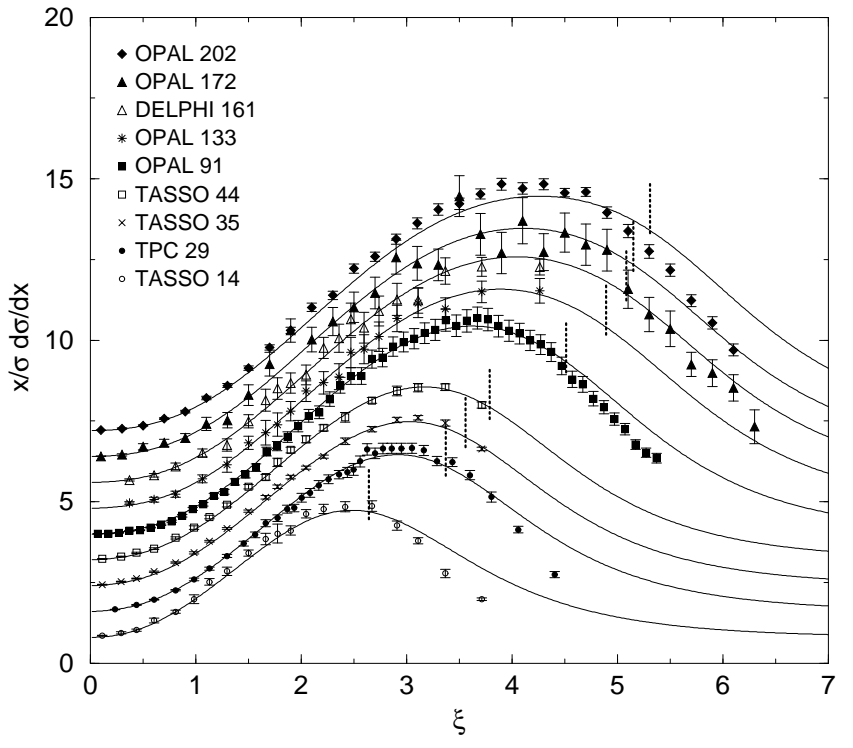

FIG. 4: Comparison with data from the fit described in Table III

TABLE IV: As in Table II but incorporating mass effects in the fit. $\Lambda_{\mathrm{QCD}}=399 \mathrm{MeV}$ and $m_{h}=252 \mathrm{MeV}$.

\begin{tabular}{|c|c|c|c|c|}
\hline $\begin{array}{ll}\mathrm{FF} & \text { Parameter }\end{array}$ & $N$ & $\beta$ & $\alpha$ & $c$ \\
\hline$g$ & 1.59 & 7.80 & -2.65 & 0.33 \\
\hline$u+c$ & 0.62 & 1.43 & {$[-2.65]$} & {$[0.33]$} \\
\hline$d+s+b$ & 0.74 & 1.60 & {$[-2.65]$} & $\begin{array}{l}{[0.33]} \\
\end{array}$ \\
\hline
\end{tabular}



FIG. 5: Comparison with data from the fit described in Table IV

both DL resummation and treatment of mass effects are required.

To extend NLO calculations to small $x_{p}$, the complete resummed DL contribution given by Eq. (52) must be added to the NLO splitting functions. These contain SGLs belonging to the classes $m=1, \ldots, 4$, which must be subtracted. Note that the NLO $m=1$ term is accounted for by the resummed DL contribution. The $m=4$ term is a type $p=0$ term, and hence does not need to be subtracted.

\section{Comparison With Gluon Jet Data}

We now compare our results with the OPAL gluon jet measurements at $E_{\text {jet }}=14.24,17.72[30]$ and $40.1 \mathrm{GeV}$ [31]. These data are shown in Fig. 6] together with our gluon FF from the fit of Table II incorporating DL resummation. The initial gluon FF from that fit is found to be about twice as large as predicted by Eq. (67), and this is reflected in the figure. In Fig. 7 we show the same



FIG. 6: Comparison of the gluon FF in the DL+LO+LO $\delta$ scheme to gluon jet measurements from OPAL. The postion of the cut of Eq. (60) is shown to indicate where the gluon is constrained. $Q=2 E_{\text {jet }}$.

plot again, but this time from a fit in which these data are included (but which is otherwise identical to the fit of Table III). The data is identified with our evolved gluon FF at $Q=2 E_{\text {jet }}$. In this case $\chi_{\mathrm{DF}}^{2}=2.3$, a slightly larger value than that from the fit of Table III in particular because the gluon jet data at $E_{\text {jet }}=14.24 \mathrm{GeV}$ cannot not be well fitted. These data give an individual $\chi_{\mathrm{DF}}^{2}$ of 3.7 , although the data at $E_{\text {jet }}=17.72$ and $40.1 \mathrm{GeV}$ give 0.9 and 1.2 respectively. The data around and beyond the peak are poorly described. The parameters and comparison with the remaining data are shown in Table $\nabla$ and Fig. 8 The value for $N_{g}$ is lower than that in Table II and in better agreement with Eq. (67). This smaller gluon FF is presumably the cause of the undershoot of 
the calculation from the data at high $\sqrt{s}$ seen in Fig. 8

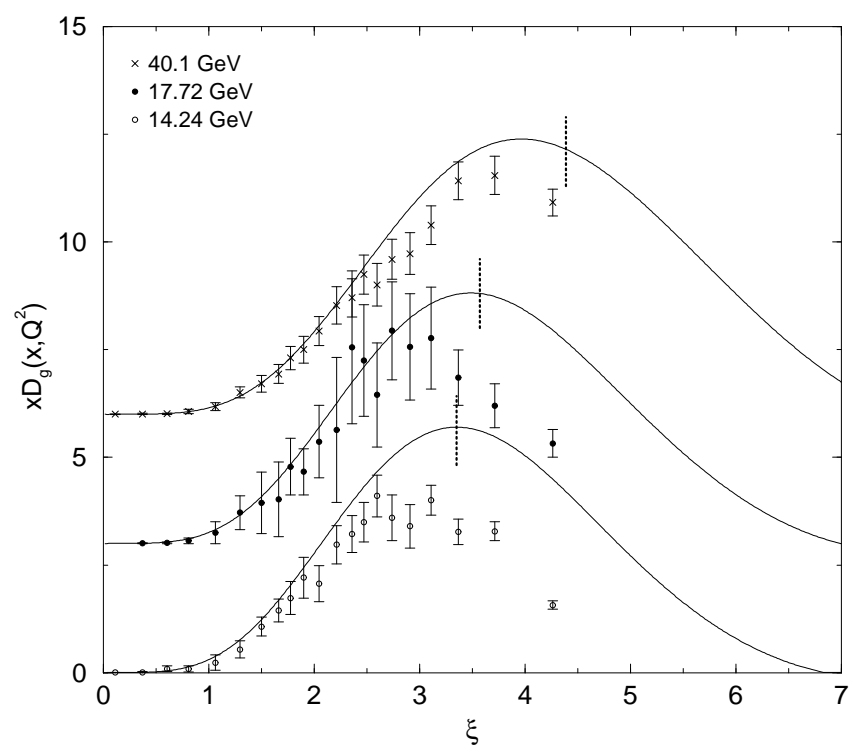

FIG. 7: As in Fig. 6 but from a fit in which the gluon jet data below the cut are included in the fit.

TABLE V: Parameter values for the FFs at $Q_{0}=14 \mathrm{GeV}$ parameterized as in Eq. (63) from a fit to all data, including the OPAL gluon jet data using DGLAP evolution in the $\mathrm{DL}+\mathrm{LO}+\mathrm{LO} \delta$ scheme. $\Lambda_{\mathrm{QCD}}=954 \mathrm{MeV}$.

\begin{tabular}{|c|c|c|c|c|}
\hline $\mathrm{FF} \quad$ Parameter & $N$ & $\beta$ & $\alpha$ & $c$ \\
\hline$g$ & 1.27 & 4.21 & -2.41 & 0.29 \\
\hline$u+c$ & 0.40 & 1.55 & {$[-2.41]$} & {$[0.29$} \\
\hline$d+s+b$ & 0.50 & 1.59 & {$[-2.41]$} & {$[0.29$} \\
\hline
\end{tabular}

Repeating the fit with hadron mass effects accounted for (including in the gluon jet data), we obtain $\Lambda_{\mathrm{QCD}}=$ $490 \mathrm{MeV}, m_{h}=302 \mathrm{MeV}$, and $\chi_{\mathrm{DF}}^{2}=2.1$. The fitted parameters, shown in Table VI are not significantly different to those of Table $\nabla$. The comparison with the data fitted to are shown in Figs. 9] and[10] The individual $\chi_{\mathrm{DF}}^{2}$ values for the gluon jet data at $E_{\text {jet }}=14.24,17.72$ and $40.1 \mathrm{GeV}$ are now $1.2,0.4$ and 0.7 respectively. We conclude that the description of the gluon jet data are also improved by including hadron mass effects, particularly at low $E_{\text {jet}}$.

In Fig. 9] we observe that the experimental data have a tendency to systematically undershoot predctions at large $\xi$. This could be partially caused by the different procedures for including soft particles in the definitions of quark and gluon jets used in the experimental analysis. We observe furthermore that these deviations decrease with increasing energy.

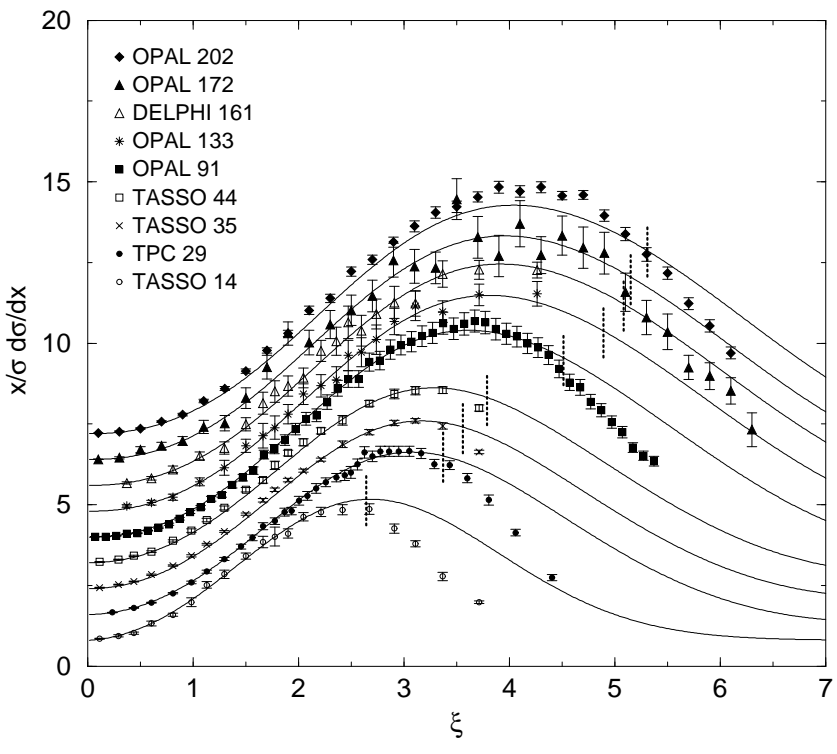

FIG. 8: Fit to data as described in Table $\nabla$



FIG. 9: As in Fig. 0 but with a fitted hadron mass.

TABLE VI: Parameter values for the FFs at $Q_{0}=14 \mathrm{GeV}$ parameterized as in Eq. (63) from a fit to all data, including the OPAL gluon jet data using DGLAP evolution in the $\mathrm{DL}+\mathrm{LO}+\mathrm{LO} \delta$ scheme and with mass effects incorporated. $\Lambda_{\mathrm{QCD}}=490 \mathrm{MeV}$.

\begin{tabular}{|c|c|c|c|c|}
\hline $\begin{array}{ll} & \text { Parameter } \\
\end{array}$ & $N$ & $\beta$ & $\alpha$ & $c$ \\
\hline$g$ & 1.30 & 5.09 & -2.30 & 0.24 \\
\hline$u+c$ & 0.46 & 1.70 & {$[-2.30]$} & {$[0.24]$} \\
\hline$d+s+b$ & 0.53 & 1.75 & {$[-2.30]$} & {$[0.24]$} \\
\hline
\end{tabular}




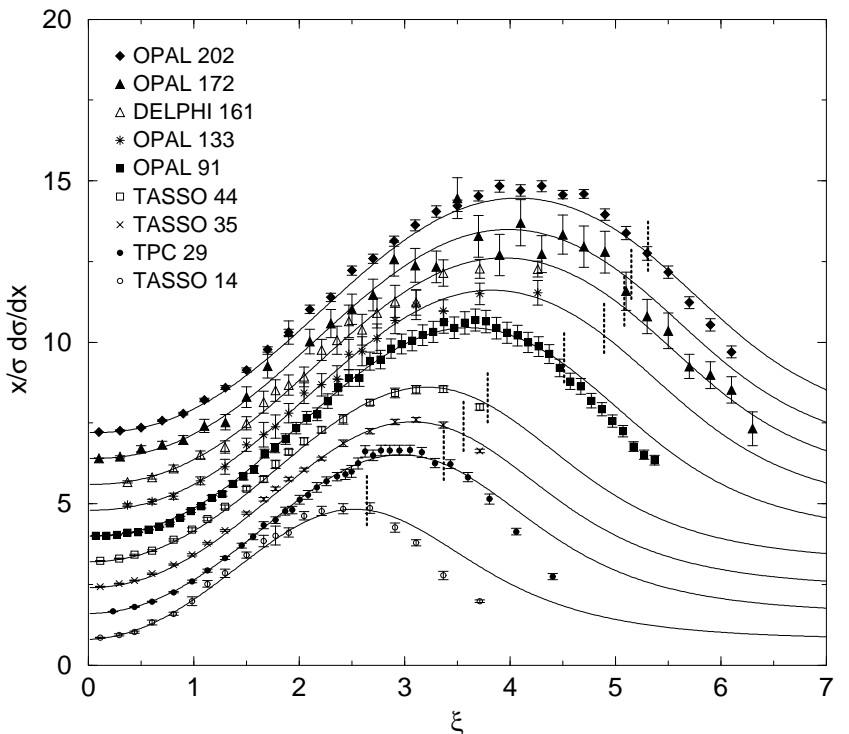

FIG. 10: Fit to data as described in Table VI

\section{CONCLUSIONS}

We have defined a general scheme for resumming SGLs in DGLAP evolution to any order in the FO contribution and to any class in the resummed SGL contribution. We have implemented it numerically at LO in the FO contribution with DL resummation, using explicit results on the LO splitting functions from the literature. This $\mathrm{DL}+\mathrm{LO}+\mathrm{LO} \delta$ scheme is obtained by taking the LO results for the splitting functions, subtracting the unresummed DLs and adding the complete resummed DL contribution, given by Eq. (52), which we obtained using the DLA. This scheme contains all contributions from the MLLA, as well as all other FO contributions at LO. We have shown by fitting to data with this scheme that for DGLAP evolution to also describe large $\xi$ data around the peak region, resummation of SGLs is necessary. In addition, we showed that both SGL resummation and treatment of hadron mass effects leads to a good fit and a reasonable result for $\Lambda_{\mathrm{QCD}}$.

In Ref. [12], it was shown that the MLLA alone allows for a good description of data around the peak region. In Ref. 9], it was shown that provided certain spurious higher order terms were removed, data above the peak region could also be well described. However, the low $\xi$ region was not well reproduced, while in Ref. [12] the good description here was due to a well known coincidence. Simultaneously fitting to both small and large $\xi$ data using either of the two approaches leads to extremely high $\chi_{\mathrm{DF}}^{2}$ values. Using the approach in this paper gives a much better fit to all the data, even if the fit is still not in the acceptable range. It allows for the data in a larger $\xi$ range to be described than the FO approach and the MLLA approach of Ref. [12] do. Further improvement in the large $\xi$ region can be expected from the inclusion of higher order SGLs.

Our scheme allows a determination of quark and gluon FFs over a wider range of data than previously achieved, and should be incorporated in NLO global fits of FFs such as that in Ref. 2] by using the method of the last paragraph of Sec. IV since the current range of $0.1<$ $x_{p}<1$ is very limited.

\section{APPENDIX A: TIMELIKE SPLITTING FUNCTIONS IN PERTURBATIVE QCD}

The LO terms of the timelike splitting functions in $x$ space for the component $D=\left(D_{\Sigma}, D_{g}\right)$ are 32 ]

$$
\begin{aligned}
P_{\Sigma \Sigma}^{(0)}(x)= & C_{F}\left(-1-x+2\left[\frac{1}{1-x}\right]_{+}+\frac{3}{2} \delta(1-x)\right), \\
P_{\Sigma g}^{(0)}= & 2 C_{F} \frac{1+(1-x)^{2}}{x}, \\
P_{g \Sigma}^{(0)}= & T_{R} n_{f}\left(x^{2}+(1-x)^{2}\right), \\
P_{g g}^{(0)}= & 2 C_{A}\left(\frac{1}{x}-2+x-x^{2}+\left[\frac{1}{1-x}\right]_{+}\right) \\
& +\left(\frac{11}{6} C_{A}-\frac{2}{3} T_{R} n_{f}\right) \delta(1-x),
\end{aligned}
$$

where $T_{R}=1 / 2, n_{f}$ is the number of flavours and, for the color gauge group $\mathrm{SU}(3), C_{A}=3$ and $C_{F}=4 / 3$. The function $[1 /(1-x)]_{+}$is defined by

$$
\begin{aligned}
& \int_{x}^{1} \frac{d y}{y}\left[\frac{1}{1-y}\right]_{+} f\left(\frac{x}{y}\right) \\
& =\int_{x}^{1} \frac{d y}{y}\left[\frac{1}{1-y}\right]\left[f\left(\frac{x}{y}\right)-y f(x)\right]+f(x) \ln (1-x)
\end{aligned}
$$

for any function $f(x)$. Transforming Eq. (A1) to Mellin space gives

$$
\begin{aligned}
P_{\Sigma \Sigma}^{(0)}(\omega)= & C_{F}\left[\frac{3}{2}+\frac{1}{(\omega+1)(\omega+2)}-2 S_{1}(\omega+1)\right], \\
P_{\Sigma g}^{(0)}(\omega)= & 2 C_{F} \frac{\omega^{2}+3 \omega+4}{\omega(\omega+1)(\omega+2)} \\
P_{g \Sigma}^{(0)}(\omega)= & T_{R} n_{f} \frac{\omega^{2}+3 \omega+4}{(\omega+1)(\omega+2)(\omega+3)} \\
P_{g g}^{(0)}(\omega)= & 2 C_{A}\left[\frac{11}{12}+\frac{1}{\omega(\omega+1)}+\frac{1}{(\omega+2)(\omega+3)}\right. \\
& \left.-S_{1}(\omega+1)\right]-\frac{2}{3} T_{R} n_{f}
\end{aligned}
$$

where, for integer $n$,

$$
S_{1}(n)=\sum_{k=1}^{n} \frac{1}{k} .
$$


The DLs and SLs at LO are obtained by expanding Eq. A3 about $\omega=0$, for which the result

$$
S_{1}(\omega+1)=1+O(\omega)
$$

is required.

The NLO splitting functions $P^{(1)}(x)$ are presented in Ref. [33], while their Mellin transforms $P^{(1)}(\omega)$ are presented in Ref. 34].

We do not explicitly present $P^{(0,1)}(x)$ for the components $D=D_{q}^{-}, D_{N S}$, since it is enough for our purposes to know that they do not contain SGLs.

\section{ACKNOWLEDGMENTS}

This work was supported in part by the Deutsche Forschungsgemeinschaft through Grant No. KN 365/51 and by the Bundesministerium für Bildung und Forschung through Grant No. 05 HT4GUA/4.
[1] B. A. Kniehl, G. Kramer and B. Pötter, Nucl. Phys. B582 (2000) 514; S. Kretzer, Phys. Rev. D62 (2000) 054001; L. Bourhis, M. Fontannaz, J. P. Guillet and M. Werlen, Eur. Phys. J. C19 (2001) 89.

[2] S. Albino, B. A. Kniehl and G. Kramer, Nucl. Phys. B725 (2005) 181.

[3] L. N. Lipatov, Yad. Fiz. 20 (1974) 181 [Sov. J. Nucl. Phys. 20 (1975) 94]; V. N. Gribov and L. N. Lipatov, Yad. Fiz. 15 (1972) 781 [Sov. J. Nucl. Phys. 15 (1972) 438]; G. Altarelli and G. Parisi, Nucl. Phys. B126 (1977) 298; Yu. L. Dokshitzer, Zh. Eksp. Teor. Fiz. 73 (1977) 1216 [Sov. Phys. JETP 46 (1977) 641].

[4] G. Curci, W. Furmanski and R. Petronzio, Nucl. Phys B175 (1980) 27; W. Furmanski and R. Petronzio, Phys. Lett. B97 (1980) 437.

[5] A. Bassetto, M. Ciafaloni, G. Marchesini and A. H. Mueller, Nucl. Phys. B207 (1982) 189; V. S. Fadin, Yad. Fiz. 37 (1983) 408 [Sov. J. Nucl. Phys. 37 (1983) 245].

[6] Y. L. Dokshitzer, V. A. Khoze, A. H. Mueller and S. I. Troian, Basics of Perturbative QCD (Editions Frontières, Gif-sur-Yvette, 1991).

[7] Y. L. Dokshitzer and S. I. Troian, Proc. 19th Winter School of the LNPI, Vol. 1, p. 144 (Leningrad, 1984); Y. L. Dokshitzer and S. I. Troian, LNPI-922 preprint (1984).

[8] A. H. Mueller, Nucl. Phys. B213 (1983) 85.

[9] S. Albino, B. A. Kniehl and G. Kramer, Eur. Phys. J. C38 (2004) 177.

[10] S. Albino, B. A. Kniehl, G. Kramer and W. Ochs, hep-ph/0503170 DESY-05-047, MPP-2005-19, Phys. Rev. Lett. (in press).

[11] J. C. Collins, Phys. Rev. D58 (1998) 094002.

[12] S. Albino, B. A. Kniehl, G. Kramer and W. Ochs, Eur. Phys. J. C36 (2004) 49.

[13] W. Braunschweig et al. [TASSO Collaboration], Z. Phys. C47 (1990) 187.

[14] M. Althoff et al. [TASSO Collaboration], Z. Phys. C22 (1984) 307.

[15] A. Petersen et al. [MARK II Collaboration], Phys. Rev. D37, (1988) 1.

[16] H. Aihara et al. [TPC/Two Gamma Collaboration], Phys. Rev. Lett. 61 (1988) 1263.

[17] R. Itoh et al. [TOPAZ Collaboration], Phys. Lett. B345
(1995) 335.

[18] ALEPH, R. Barate et al. [ALEPH Collaboration], Phys. Rept. 294 (1998) 1.

[19] DELPHI, P. Abreu et al. [DELPHI Collaboration], Z. Phys. C73 (1996) 11.

[20] B. Adeva et al. [L3 Collaboration], Phys. Lett. B259 (1991) 199.

[21] M. Z. Akrawy et al. [OPAL Collaboration], Phys. Lett. B247 (1990) 617.

[22] G. S. Abrams et al. [SLC Collaboration], Phys. Rev. Lett. 64 (1990) 1334.

[23] D. Buskulic et al. [ALEPH Collaboration], Z. Phys. C73 (1997) 409.

[24] G. Alexander et al. [OPAL Collaboration], Z. Phys. C72 (1996) 191.

[25] K. Ackerstaff et al. [OPAL Collaboration], Z. Phys. C75, (1997) 193.

[26] G. Abbiendi et al. [OPAL Collaboration], Eur. Phys. J. C16 (2000) 185.

[27] G. Abbiendi et al. [OPAL Collaboration], Eur. Phys. J. C27 (2003) 467.

[28] S. Lupia and W. Ochs, Eur. Phys. J. C2 (1998) 307.

[29] Y. I. Azimov, Y. L. Dokshitzer, V. A. Khoze and S. I. Troian, Z. Phys. C31 (1986) 213.

[30] G. Abbiendi et al. [OPAL Collaboration], Phys. Rev. D69 (2004) 032002.

[31] G. Abbiendi et al. [OPAL Collaboration], Eur. Phys. J. C11 (1999) 217.

[32] G. Altarelli and G. Parisi, Nucl. Phys. B126 (1977) 298.

[33] G. Curci, W. Furmanski and R. Petronzio, Nucl. Phys. B175 (1980) 27; W. Furmanski and R. Petronzio, Phys. Lett. B97 (1980) 437; E. G. Floratos, C. Kounnas and R. Lacaze, Nucl. Phys. B192 (1981) 417; J. Binnewies, B. A. Kniehl and G. Kramer, Z. Phys. C76 (1997) 677; R. K. Ellis, W. J. Stirling and B. R. Webber, "QCD and collider physics," Camb. Monogr. Part. Phys. Nucl. Phys. Cosmol. 8 (1996) 1, Chapter 6. In the latter reference, some misprints from the earlier publications are corrected.

[34] M. Gluck, E. Reya and A. Vogt, Phys. Rev. D48 (1993) 116 [Erratum-ibid. D51 (1995) 1427]. 